\title{
Diagnostic performance of diffusion-weighted magnetic resonance imaging in pulmonary malignant lesions: a meta-analysis
}

\author{
Ning Chang ${ }^{1 \#}$, Xiao-Hui Wang ${ }^{2 \#}$, Long-Biao Cui ${ }^{3}$, Hong Yin ${ }^{3}$, Tao Jiang ${ }^{4}$, Fu-lin Chen ${ }^{5}$ Li-Peng Liu ${ }^{1,6}$, \\ Jian Zhang ${ }^{1}$
}

${ }^{1}$ Department of Pulmonary and Critical Care Medicine, Xijing Hospital, Fourth Military Medical University, Xi'an 710032, China; ${ }^{2}$ Department of Pulmonary and Critical Care Medicine, The First Affiliated Hospital of Chongqing Medical University, Chongqing 400016, China; ${ }^{3}$ Department of Radiology, Xijing Hospital, Fourth Military Medical University, Xi'an 710032, China; ${ }^{4}$ Department of Medical Oncology, Shanghai Pulmonary Hospital, Tongji University School of Medicine, Shanghai 710032, China; ${ }^{5}$ College of Life Sciences, Northwest University, Xi'an 710069, China; ${ }^{6}$ Division of Pediatric Blood Diseases Center, Institute of Hematology and Blood Diseases Hospital, Chinese Academy of Medical Sciences \& Peking Union Medical College, Tianjin 300020, China

Contributions: (I) Conception and design: N Chang, XH Wang, LP Liu, J Zhang; (II) Administrative support: LP Liu, J Zhang; (III) Provision of study materials or patients: LB Cui; (IV) Collection and assembly of data: LB Cui, H Yin; (V) Data analysis and interpretation: LP Liu; (VI) Manuscript writing: All authors; (VII) Final approval of manuscript: All authors.

"These authors contributed equally to this work.

Correspondence to: Li-Peng Liu; Jian Zhang. Department of Pulmonary and Critical Care Medicine, Xijing Hospital, Fourth Military Medical University, Xi'an 710032, China. Email: lipeng88859@163.com; 376644181@qq.com.

Background: Overuse or misuse of positron emission tomography/computed tomography (PET/CT) should be avoided for its ionizing-radiation. Diffusion-weighted magnetic resonance imaging (DW-MRI), characterized by no radiation, may be regarded as an alternative in differentiating pulmonary nodules. We aim to estimate the diagnostic accuracy of DW-MRI in diagnosing of pulmonary lesions.

Methods: Relevant studies were searched through PubMed and Embase with no language restriction from inception to March 8, 2019. We selected studies reporting sensitivity and specificity of DW-MRI for differentiating pulmonary nodules. A summary estimates of sensitivity, specificity and area under curve (AUC) of receiver operating characteristic (ROC) of DW-MRI were analyzed with a random effects model.

Results: We included data from 37 studies, which altogether included 2,311 pulmonary lesions. The pooled sensitivity and specificity were 0.86 (95\% CI, 0.82-0.89) and 0.79 (95\% CI, 0.72-0.85), and AUC was 0.90 (95\% CI, 0.87-0.92). Subsequent subgroup analysis showed the higher sensitivity of DW-MRI in pulmonary lesion $>2 \mathrm{~cm}$ in comparison to lesions $\leq 2 \mathrm{~cm}$, however, higher specificity was observed in smaller lesions.

Conclusions: Radiation-free DW-MRI showed a favorable balance between sensitivity and specificity in diagnosing pulmonary malignancies especially in lesion size $\leq 2 \mathrm{~cm}$. Existing evidence indicated that DWMRI may be considered as an independent substitute in diagnosis of lung lesions, which might help to prevent long-term side-effects from radiographic diagnosing and evaluating procedures.

Keywords: Pulmonary malignant lesions; pulmonary nodules; diffusion-weighted magnetic resonance imaging; diagnostic; meta-analysis

Submitted Jul 05, 2019. Accepted for publication Sep 09, 2019.

doi: $10.21037 /$ tlcr.2019.10.08

View this article at: http://dx.doi.org/10.21037/tlcr.2019.10.08 


\section{Introduction}

Although ${ }^{18} \mathrm{~F}$-fludeoxyglucose (FDG) positron emission tomography (PET) is recommended to evaluate suspicious lesions by diagnostic guidelines (1-3), the American Society of Clinical Oncology (ASCO) and European Society for Medical Oncology (ESMO) have all claimed that PET or PET/computed tomography (PET/CT) had been overused or misused and declined to include surveillance PET or PET/CT in disease-specific guidelines (4). For instance, US annual per capita radiation dose increased from $0.1 \mathrm{mSv}$ in 1980 to $0.77 \mathrm{mSv}$ in 2006 from the source of nuclear medicine (5).

However, there have not been radiation-free and noninvasive test recommended by guideline used for diagnosis and evaluation in cancer patients so far. With consideration of this condition, magnetic resonance imaging (MRI) has been gaining increasing attention due to its radiation-free characteristic. There are several well controlled and rigorously conducted investigations showing that diffusion-weighted MRI (DW-MRI) could achieve a comparable even better performance in cancer diagnosis and metastasis staging as compared to PET/CT (6-11). In clinical practice, however, clinicians generally only consider PET or PET/CT as the method used for cancer staging and follow-up examination, but ignore the potential utilization of DW-MRI in cancer patients. As for DW-MRI, it is cost- and time-efficient, and no contrast agent is involved during screening, but if it could be regarded as an alternative selection to diagnose pulmonary lesions is still to be confirmed.

A recent meta-analysis by Deepen et al. have found PET combined with CT or not with reduced specificity of $61 \%$ in regions where infectious lung disease is endemic (12). MRI yields information, such as integrity, about microscopic structures. When analyzing quantitatively in DW-MRI, there is a significant difference in apparent diffusion coefficient (ADC) values between malignant and benign lesions. Most recently, Shen et al. reported ADC value was helpful for distinguishing malignant and benign lung lesions (13). In the only known meta-analysis about accuracy of DWMRI in lung lesions, this modality was reported to be useful for differentiation between malignant and benign pulmonary lesions with pooled sensitivity of $84 \%$ and specificity of $84 \%$ (14). However, no standardization of quality assurance protocols for DW-MRI was included in their study, which was critical to repeatability of this imaging modality in cancer screening. ADC was merely focused on as an imaging measure for lung lesion diagnosis in the previous meta-analysis by Shen et al., lesion size was not considered and only 10 studies were included in the study (13). In view of this, no sufficient evidence has been available to firmly establish the advantages of DW-MRI test performance to diagnose pulmonary malignancies so far.

Our aim was to estimate the sensitivity, specificity, diagnostic odds ratio (DOR) and area under receiveroperator characteristics curve (AUC) of DW-MRI for discrimination between malignant and benign pulmonary lesions. Moreover, we intended to clarify the lesion size and other indispensable parameters of DW-MRI which may affect the accuracy of DW-MRI and drawing attention of researchers in the future.

\section{Methods}

We did a meta-analysis in accordance with the Preferred Reporting Items for Systematic reviews and Meta-Analyses (PRISMA) guidelines and the guidelines described in the Cochrane Handbook for Systematic Reviews of Diagnostic Test Accuracy (15).

\section{Search strategy and selection criteria}

We searched Embase and PubMed to identify the relevant studies. Under the supervision of a librarian at the Fourth Military Medical University, we searched these databases from their inception through March 8 2019. No language restriction was placed on these searches. We checked reference lists of all retrieved articles to identify additional suitable studies. A radiologist and an oncologist were asked to look through these literatures and assess their eligibility for analysis. The inclusion criteria included studies that assessed the diagnostic accuracy of DW-MRI in lung lesions. The inclusion criteria included: (I) studies that assessed the diagnostic accuracy of DW-MRI, among which systematic reviews and meta-analyses were used only as a source of references, (II) studies that assessed pulmonary lesions, (III) studies that validated the performance of DW-MRI in lung lesion diagnosis and should state that all participants had the index and reference tests, and (IV) studies which was possible to allow calculation of sensitivity and specificity. Conference abstracts were included when they contained relevant data or relevant unpublished data could be obtained from the authors. We excluded all studies that could be classified as (I) narrative reviews, letters, 
editorials, comments, and case reports; (II) surveillance of the tumor response to therapy and survival in patients with cancers treated with chemoradiotherapy. A total of 37 studies were finalized. Any disagreement between them was resolved by discussing with a third investigator. The information, including author list, journals, affiliation, and the publication date, remained blinded to the above reviewers. All studies were selected in two rounds, first on title and abstract and second on full text, against the following criteria.

\section{Quality assessment}

The quality of the selected studies and the potential bias were assessed using the pre-specified STARD and the Quality Assessment of Diagnostic Accuracy Studies (QUADAS-2) instrument (16), including additional items as recommended by the Cochrane Collaboration. This quality assessment procedure was independently performed by two pairs of reviewers and was checked by a fifth reviewer. Any disagreements were resolved by discussion involving all researchers when necessary. The reference of standard (ROS) was validated by a clinical review committee consisting of three researchers.

\section{Data extraction}

Two reviewers independently extracted relevant data from the selected studies in a standard form, a third investigator checked the extracted data, and a fourth investigator arbitrated on discrepancies between the first two investigators. Any identified discrepancies were discussed and corrected. $2 \times 2$ contingency tables were constructed, summarizing true positives (TP), false positives (FP), true negatives (TN), and false negatives (FN). Moreover, if various kinds of research type (per-patient $v s$. per-lesion and DW-MRI only vs. DW-MRI with other sequence) were available in individual study, we incorporated them into our study separately and made a subgroup analysis of each type. In some investigations, either the number of patients or the number of lesions was used for the statistical analyses. In order to avoid this inconsistence, we also conducted separate analyses for each category.

\section{Statistical analysis}

A random effects model was performed for the primary meta-analysis using a non-linear mixed model approach.
The objective is to estimate the sensitivity and the specificity with $95 \%$ confidential intervals (CIs) of DW-MRI. We also computed the distribution of individual studies on summary receiver-operator characteristic (sROC) plots. Positive and negative likelihood ratios $\left(\mathrm{LR}^{+}\right.$and $\left.\mathrm{LR}^{-}\right)$are metrics that combine sensitivity and specificity in their calculation for the discriminating ability of each imaging modality $(17,18)$. If the $\mathrm{LR}^{+}$is $>5.0$ and the $\mathrm{LR}^{-}$is $<0.2$, then the test can both rule in and rule out the disease.

The heterogeneity was assessed using the Cochrane Q and $\mathrm{I}^{2}$ statistics (19). $\mathrm{I}^{2}$ over $50 \%$ indicates heterogeneous, while $\mathrm{P}<0.05$ was considered having heterogeneity in likelihood ratio $\chi^{2}$ test. We assessed publication bias by Deeks' plots (20). Subgroup analyses by statistical modeling were planned for pre-specified items. Allowing for variation in result of three signaling questions in QUADAS-2, we put them into subgroup analysis (consecutive enrollment, reference standard and operation interval) to seek potential impact. To assess the impact of covariates (i.e., subgroup factors) on test performance of DW-MRI for cancer diagnosing, we considered a meta-regression with various covariates, and it is a tool used in meta-analysis to examine the impact of moderator variables on study effect size using regression-based techniques. It is more effective at this task than standard meta-analytic techniques (21).

We reported the average adjusted estimates of sensitivity and specificity because of the validity of their interpretation and general applicability. All tests were 2-sided with a type I error of .05. All analyses were performed using the software StataSE version 12 (StataCorp).

\section{Results}

A total of 748 articles were reviewed. Among them, 711 were excluded after primary and subsequent reviewing. The remaining 37 articles involved 2,311 pulmonary lesions (Figure 1, Table S1).

The quality of the included studies was assessed by the QUADAS-2 tool. Discriminations were primarily found in domain of "consecutive enrollment, reference standard and operation interval" for all studies. Consequently, we selected these signaling questions as covariates in subgroup analysis, to explore if they could affect the accuracy of our research.

For the assessment of efficacy of DW-MRI in lung cancer (with a 95\% CI reported in the included individual studies), the detailed sensitivity and specificity values were illustrated by forest plot (Figure 2). Pooled sensitivity and specificity of 37 studies for DW-MRI were 0.86 (95\% CI, 


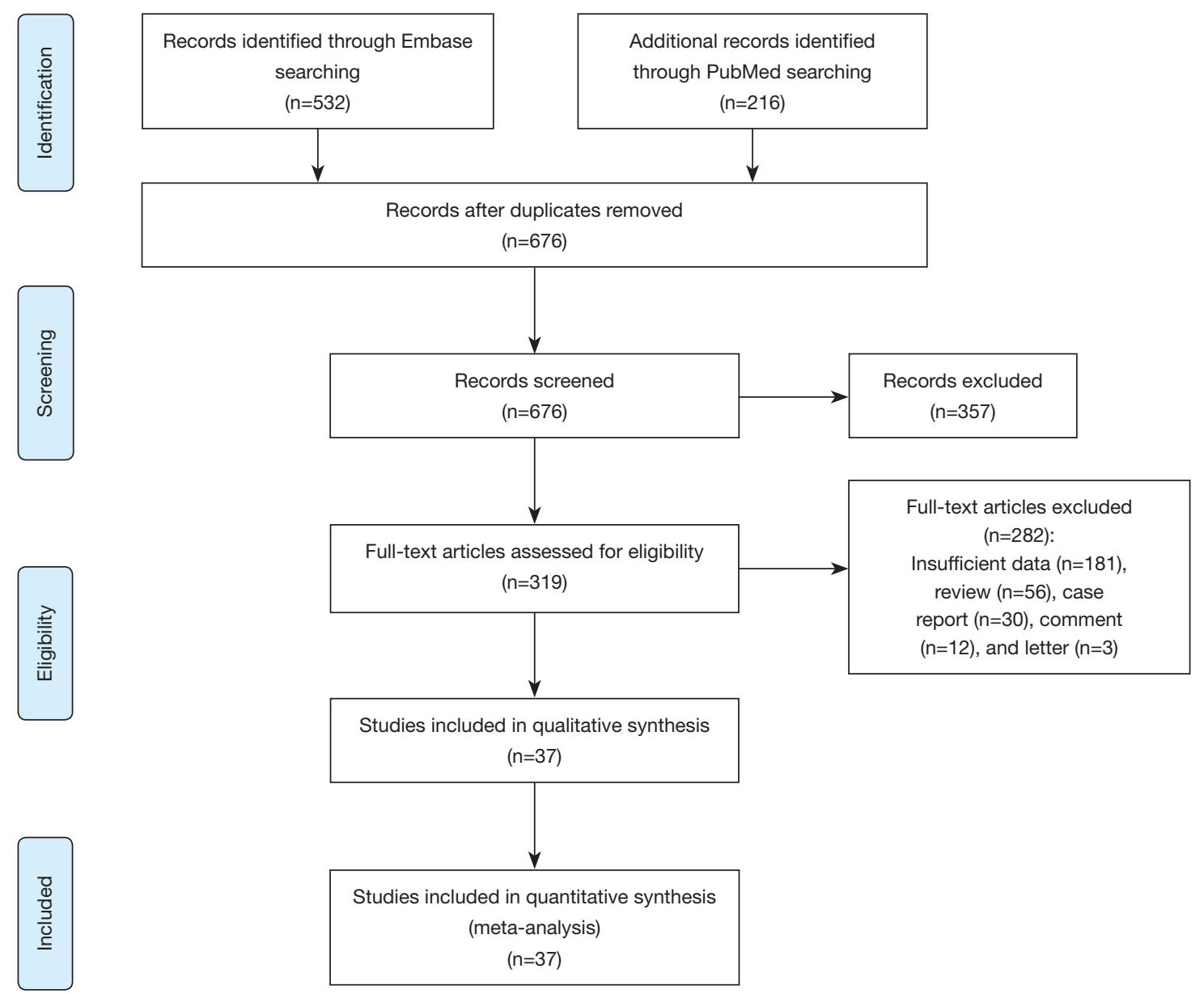

Figure 1 Flowchart for the selection procedure for studies.

$0.82-0.89$ ) and 0.79 (95\% CI, 0.72-0.85). The value of diagnostic odds ratio (DOR) was 23 (95\% CI, 15-36), while summary estimates were 4.1 (95\% CI, 3.1-5.6) for positive likelihood ratio $\left(\mathrm{LR}^{+}\right)$and 0.18 (95\% CI, 0.14-0.23) for negative likelihood ratio $\left(\mathrm{LR}^{-}\right)$. All these results indicated DW-MRI's excellent ability to both confirm and exclude presence of lung cancer.

Meta-regression showed heterogeneity from DWMRI examinations were found in subgroups of $b$ value $\left(\mathrm{I}^{2}=0, \mathrm{P}=0.48\right)$. There was also a trend for the subgroup of lesion size to show heterogeneity $\left(\mathrm{I}^{2}=98, \mathrm{P}<0.01\right)$. Allow for lesion size is a heterogeneous factor which may affect the diagnostic accuracy of screening modality, a new histopathological classification for pulmonary nodule was proposed measuring $\leq 2 \mathrm{~cm}$ and $>2 \mathrm{~cm}$ in maximum dimension. Among the included studies, 14 reported average or median lesion size of less than $2 \mathrm{~cm}$, and 16 larger than $2 \mathrm{~cm}$. Average adjusted sensitivity of DW-MRI to diagnose lung cancer was significantly influenced by lesion size $(0.83$, (95\% CI, 0.80-0.85) for studies with mean lesions $\leq 2 \mathrm{~cm}$ and 0.86 (95\% CI, 0.83-0.88) for lesion size $>2 \mathrm{~cm} \mathrm{P}<0.01$ ), however, results indicated that average adjusted specificity of lesion size $\leq 2 \mathrm{~cm}(0.85 ; 95 \%$ CI, $0.80-0.89)$ significantly higher than lesion size $>2 \mathrm{~cm}(0.77 ; 95 \% \mathrm{CI}, 0.71-0.82)$ $(\mathrm{P}<0.01)$.

The sROC curves reported the predictive value of DWMRI for all studies, the AUC value was 0.90 (95\% CI, 0.87-0.92) (Figure 3). The ROC space did not illustrate a curvilinear trend of points and no threshold effect in diagnostic accuracy was observed. Since we tried to gather all evidence in the published works, potential biases were unavoidable. The Deeks' funnel plot showed the evidence of publication bias towards studies $(\mathrm{P}<0.05)$ (Figure 4). In view of this, we performed the subgroup analysis without this study and estimated the publication bias for each subgroup of different lesion size, asymmetric test did not 

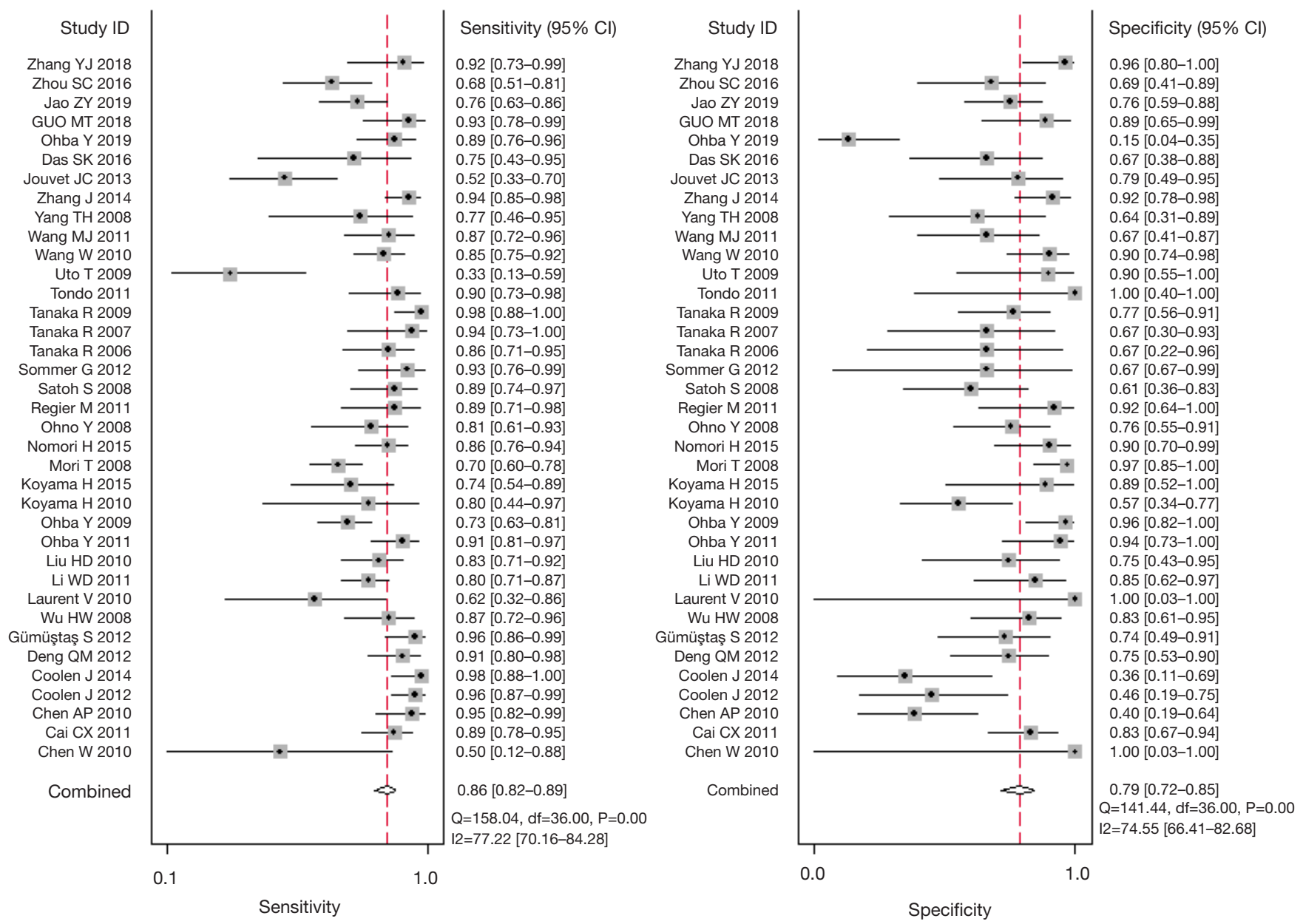

Figure 2 Forest plots of sensitivity and specificity for DW-MRI in 37 studies.

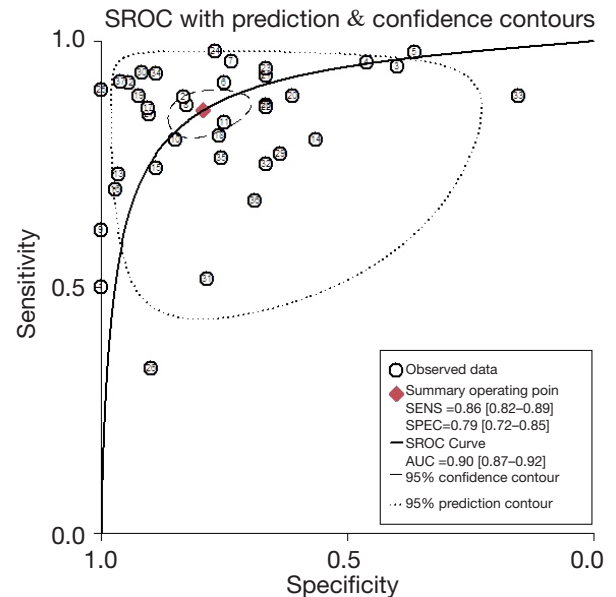

Figure 3 Summary receiver-operator characteristic (sROC) curves for DW-MRI in 37 studies. show any evidence in lesion size $>2 \mathrm{~cm}(\mathrm{P}=0.09)$ and $\leq 2 \mathrm{~cm}$ $(\mathrm{P}=0.14)$ (Figures 5,6).

Table 1 illustrated average adjusted sensitivity and specificity in subgroup analysis to estimate the magnitude of the effect by classifying studies in each covariate. As for critical parameter, DW-sequences are now almost routinely used as an adjunct to conventional MRI images. However, if it requires any other morphologic sequences to improve accuracy? The adjusted sensitivity and specificity for the imaging modality with DWI sequence only was 0.84 (95\% CI, 0.78-0.90) and 0.75 (95\% CI, 0.67-0.82), while those for DWI combined with other sequence was 0.84 (95\% CI, $0.79-0.89$ ) and 0.77 (95\% CI, 0.69-0.86), the pooled AUC was 0.87 and 0.91 for DWI sequence only and DWI with other sequence. Our results indicated that accuracy of DWI sequence only was lower than that of DWI combined with 


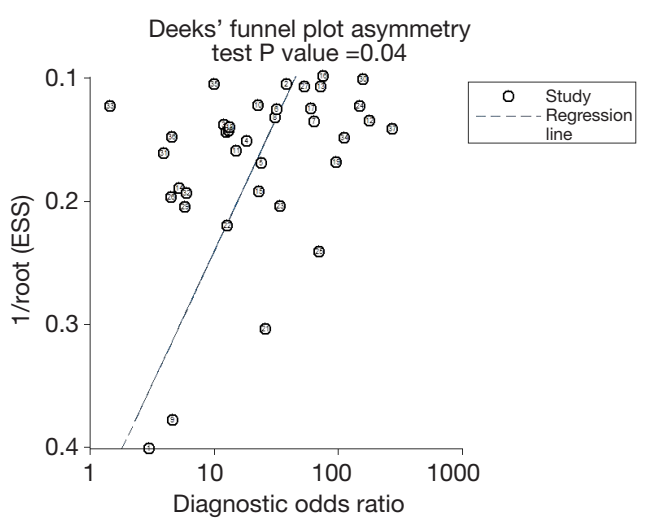

Figure 4 The Deeks' funnel plot showed publication bias towards studies.

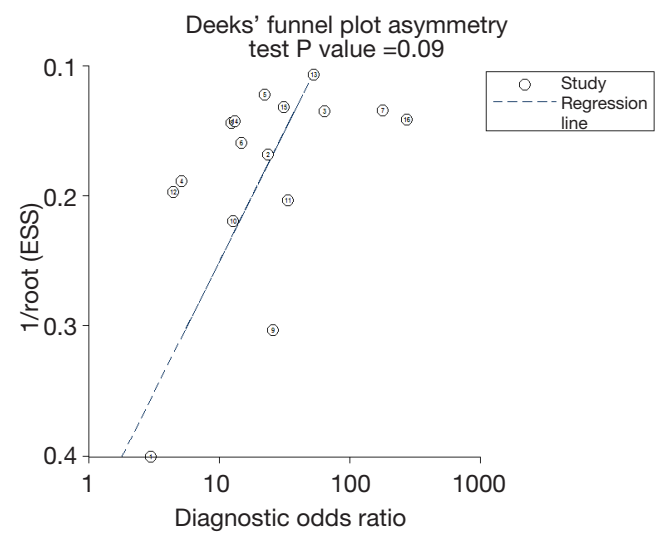

Figure 5 The Deeks' funnel plot showed the publication bias in lesion size $>2 \mathrm{~cm}$.

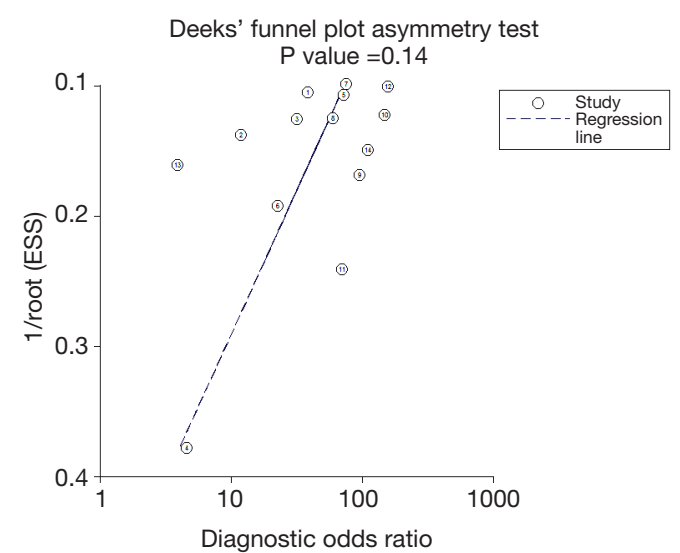

Figure 6 The Deeks' funnel plot showed the publication bias in lesion size $\leq 2 \mathrm{~cm}$. other sequence.

Additionally, DW-MRI should be performed with sufficient degrees by appropriate choices of $b$ values, with considerations given for the anatomic region, tissue composition, and pathologic processes. According to previous study, we summarized b values that may be used as a guide when performing DW-MRI for qualitative assessment and $750-1,000 \mathrm{~s} / \mathrm{mm}^{2}$ may be served as optimal value for wholebody imaging purpose. Thus, we conducted a subgroup analysis to compare the $b$ value of $750-1,000 \mathrm{~s} / \mathrm{mm}^{2}$ with other values, pooled sensitivity, specificity and AUC of b value of $750-1,000 \mathrm{~s} / \mathrm{mm}^{2}$ was 0.84 (95\% CI, 0.79-0.88), 0.79 (95\% CI, 0.72-0.86) and 0.91, while those of other b value were comparatively lower of 0.84 (95\% CI, 0.78-0.91), 0.71 (95\% CI, 0.58-0.85) and 0.88, respectively. Field strength is another essential parameter of MRI in predicting lung lesion. On the basis of our result, although $1.5 \mathrm{~T}$ was less sensitive than $3.0 \mathrm{~T}$ in screening pulmonary nodule [0.82 (95\% CI, 0.77-0.87) vs. 0.88 (95\% CI, 0.83-0.92)], it was more specific than $3.0 \mathrm{~T}[0.80$ (95\% CI, $0.74-0.86 \mathrm{vs}$. 0.69 (95\% CI, 0.53-0.85)].

As for study method, the adjusted sensitivity and specificity for quantitative analysis was 0.84 (95\% CI, $0.79-0.89)$ and 0.79 (95\% CI, 0.71-0.87), while those for qualitative analysis was 0.84 (95\% CI, 0.78-0.90) and 0.73 (95\% CI, 0.63-0.83). It was significantly specific in quantitative analysis $(\mathrm{P}<0.05)$. What's more, prospective design and consecutive enrollment were with higher sensitivity in comparison to retrospective and inconsecutive study design.

\section{Discussion}

Use of PET/CT for routine surveillance is now clearly not recommended by Centers for Medicare and Medicaid Services (CMS) (22). In concurrence with this assessment, Cancer Care Ontario systematically reviews the literature and to date has not recommended PET/CT for surveillance (23). Available evidence from clinical studies suggests that using PET/CT to monitor for recurrence does not improve outcomes and therefore generally is not recommended, notably increased radiation from PET and PET/CT (5). Until high-level evidence demonstrates that routine surveillance with PET/ CT scans help prolong life or promote well-being after treatment for a specific type of cancer, this practice should 


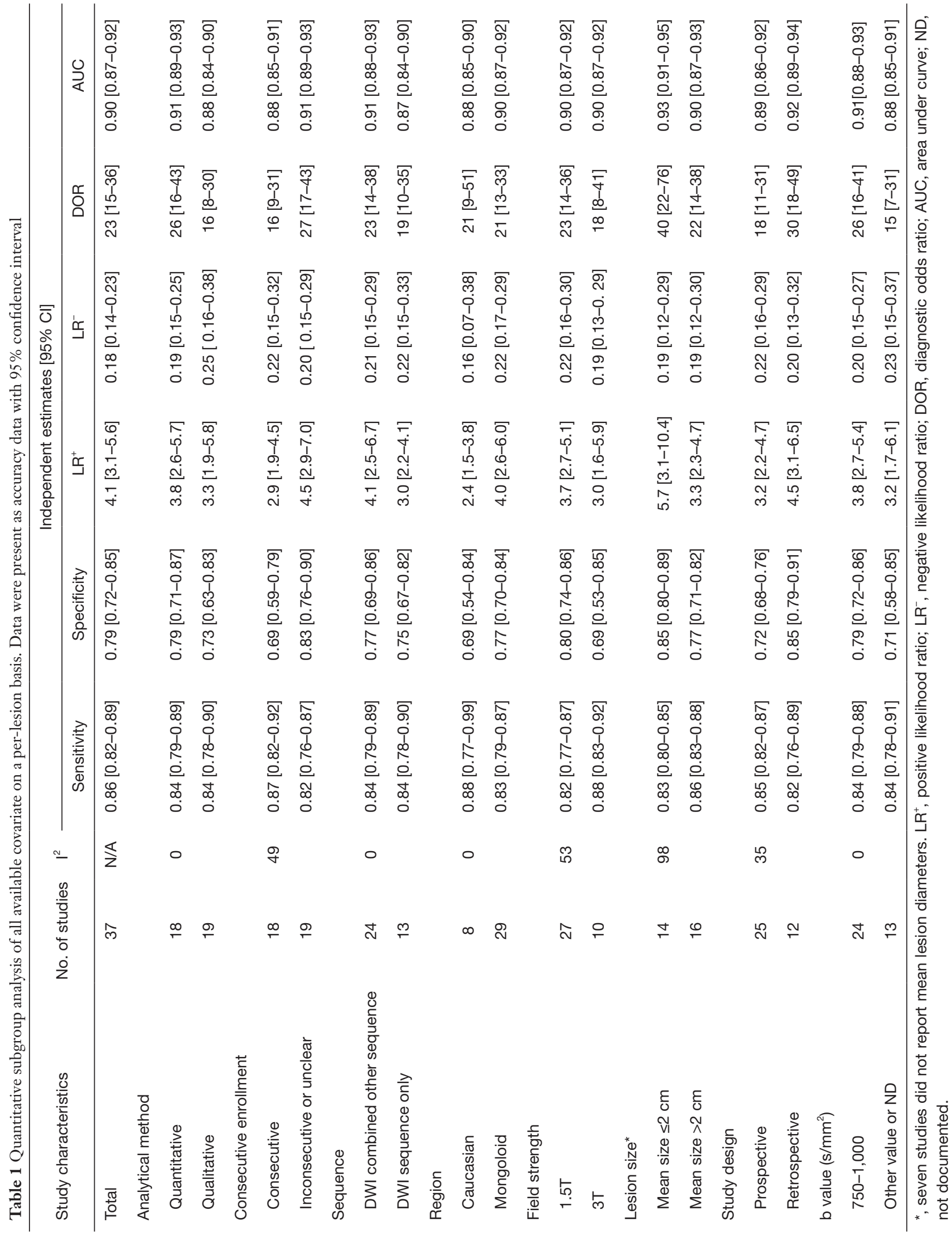


not be performed (4). Therefore, if radiation-free DW-MRI could be an alternative selection, this imaging test might help to prevent long-term side-effects from radiographic staging procedures.

Substantial advantage lies in the new MRI techniques nowadays. The principle of DW-MRI exploits the random motion, Brownian movement, of water protons in biologic tissue, leading to DW-MRI possessing no exposure to ionizing radiation and reflecting the diffusivity of water molecules in tissue. When ADC values are assessed with DW-MRI, there is a significant difference in ADC between malignant and benign lesions. Moreover, the use of T1weighted sequence obviates the need for an intravenous contrast medium, so patients with poor renal function can also undergo MRI for this purpose. In addition, compared to PET or PET/CT, advantages of DW-MRI include no anaphylaxis to high spatial resolution of images, diverse information from various sequences, high efficiency, and low consumption. To some extent these might explain why DW-MRI may outperform PET/CT in the assessed contexts.

Pulmonary lesion is a common finding on chest imaging, in which lung cancer represents the most frequently diagnosed malignancy. Lung cancer is regarded as the most common cancer and leading cause of cancer death in the whole world $(24,25)$. Radiation-free DW-MRI, which has been applied in recent years, may yields similar or even superior diagnostic value especially in lung cancer in comparison of PET/CT (10). The combination of anatomical, physiological and biological information makes multiparametric MRI an appealing tool for the diagnosis of malignant lesions, among which DW-MRI, visualizing the diffusion characteristics of tissue, is widely used and has presented promising results. Since the diagnostic performance of DW-MRI in distinguishing pulmonary lesions has been performed in many studies, we attempt to evaluate the overall diagnostic accuracy with a metaanalysis.

We observed lower sensitivity but higher specificity of DWMRI in diagnosis of pulmonary malignancies when compared with sensitivity of 0.89 (95\% CI, 0.87-0.91) and specificity of 0.75 (95\% CI, 0.71-0.78) in PET/CT on basis of a recent study by Deppen et al. (12). Allow for heterogeneous results in different lesion size of imaging modality, we compared average adjusted sensitivity and specificity between DW-MRI and PET/CT in Deppen's study, which nodule was proposed measuring $\leq 2 \mathrm{~cm}$ and $>2 \mathrm{~cm}$ in maximum dimension. In comparison, DW-MRI had a significantly higher average adjusted specificity in both $\leq 2 \mathrm{~cm}$ and $>2 \mathrm{~cm}$ subgroup, although with lower sensitivity $(\mathrm{P}=0.01$ for studies with lesions $\leq 2 \mathrm{~cm}$ and $\mathrm{P}<0.01$ for studies with larger average lesion size) in comparison to PET/CT in both subgroup. Although adjusted specificity of DW-MRI was higher than PET/CT, we found it had a lower specificity among studies reporting larger lesions than those with smaller lesions. From the practical aspect, difficulty of defining areas where the regions of interest are made to measure $\mathrm{ADC}$ values due to variable signal intensity on ADC maps, leading to certain confounds, might account for lower specificity in larger lesions.

As a trend in clinical practice, parameters in examination modalities were increasingly added to analytical method. Empirically, our study showed quantitative analysis with $A D C$ value was with similar sensitivity and higher specificity than qualitative analysis in differentiating pulmonary lesion, which may imply that $\mathrm{ADC}$ is an objective value that can improve the efficiency of DW-MRI in screening on pulmonary nodules. Most scanners generate identical $\mathrm{ADC}$ value without significant difference, regardless of the manufacture, scanner type, field strength, gradient strength, or gradient slew rate. There are agreements among all stakeholders on standards for both acquisition protocols, repeatability/reproducibility and for the post-processing procedures, to ensure that quantitative $\mathrm{ADC}$ values have similar meanings across institutions, because the technique is quantifiable and can be repeated easily. For instance, the measured ADC values show good reproducibility between different MR systems, a GE 1.5 T (Signa TwinSpeed HD, GE Healthcare, Milwaukee, WI), a Siemens 1.5 T (Magnetom Espree, Siemens Healthcare, Erlangen, Germany) and a Philips 3.0 T (Achieva Dual, Philips Healthcare, Best, The Netherlands) scanners (26). Thus, $\mathrm{ADC}$ value has the potential for clinical trials and cancer screening.

As for standardized data sets, additionally, DW-MRI should be performed with sufficient degrees by appropriate choices of $\mathrm{b}$ values, with considerations given for the anatomic region, tissue composition, and pathologic processes. According to a previous study, we summarized b values that may be used as a guide when performing DW-MRI for qualitative assessment and $750-1,000 \mathrm{~s} / \mathrm{mm}^{2}$ may be served as optimal value for whole-body imaging purpose. Thus, we conducted a subgroup analysis to compare the $b$ value of $750-1,000$ with other values, and the results confirmed that $\mathrm{b}$ value of $750-1,000 \mathrm{~s} / \mathrm{mm}^{2}$ was with comparable sensitivity and significant higher specificity in comparison to others. It may 
be concluded that $750-1,000 \mathrm{~s} / \mathrm{mm}^{2}$ may be served as optimal value for DW-MRI in whole-body imaging purpose.

Although subgroup of prospective and consecutive enrollment only exerted higher sensitivity, it may be caused by limited sample size or other potential biases. On basis of our result, we suggest more scientific experiment design in future, which will predict the efficacy of imaging modality in a more convincing way. Despite a noninvasive test to assess the risk of cancer or benign disease recommended by diagnostic guidelines (1-3), PET or PET/CT should be limited for use of diagnosing lung cancer in regions where infectious lung disease is endemic (12). Our meta-analysis established the advantages of DW-MRI for the diagnosis of lung cancer. This strong evidence exists for health-care systems to consider the introduction of DW-MRI as a crucial evaluating examination for lung lesions.

Advances in MRI technique, providing operability to achieve whole-body DW imaging and excellent tissue contrast, have led to good diagnostic performance in clinical practice and investigation. DW-MRI, for either wholebody or regional purposes $(7,8)$, is no more technically challenging and prone to be reliable as an examination.

Although the application of PET/CT has grown rapidly during the past several years, this modality is associated with substantial exposure to ionizing radiation equivalent to roughly 700-750 chest radiographs (27). Exposure to ionizing radiation in radiosensitive patients causes roughly an increased risk of cancer, especially leukemia and brain cancer (28). According to our result, radiation-free DWMRI may be considered as a potential alternative by medical practitioner. This finding will contribute to balance against an increasing tendency of diagnosing cancer patient using PET/CT initially and the risk of radiation on certain population before evaluation has been done.

\section{Conclusions}

Radiation-free DW-MRI shows a favorable balance between sensitivity and specificity in diagnosing pulmonary malignancies. Existing evidence may indicate that DWMRI could be considered as an independent substitute in diagnosis of lung lesions, which might help to prevent long-term side-effects from radiographic diagnosing and evaluating procedures.

\section{Acknowledgments}

None.

\section{Footnote}

Conflicts of Interest: The authors have no conflicts of interest to declare.

Ethical Statement: The authors are accountable for all aspects of the work in ensuring that questions related to the accuracy or integrity of any part of the work are appropriately investigated and resolved.

\section{References}

1. MacMahon H, Austin JH, Gamsu G, et al. Guidelines for management of small pulmonary nodules detected on CT scans: a statement from the Fleischner Society. Radiology 2005;237:395-400.

2. Yan J, Wu Z, Li S. Extremely low dose 18F-FDG PET imaging and its potential use for lung cancer screening. Transl Cancer Res 2017;6:S99-101.

3. Jie Y, Meng X, Gu A, et al. Metabolic volume parameters based on different thresholds with baseline 18F-FDG PET/CT as prognostic factors for survival in stage III non-small cell lung cancer. Transl Cancer Res 2017;6:732-45.

4. Schnipper LE, Lyman GH, Blayney DW, et al. American Society of Clinical Oncology 2013 top five list in oncology. J Clin Oncol 2013;31:4362-70.

5. Linet MS, Slovis TL, Miller DL, et al. Cancer risks associated with external radiation from diagnostic imaging procedures. CA Cancer J Clin 2012;62:75-100.

6. Lichy MP, Aschoff P, Plathow C, et al. Tumor detection by diffusion-weighted MRI and ADC-mapping--initial clinical experiences in comparison to PET-CT. Invest Radiol 2007;42:605-13.

7. Ohno Y, Koyama H, Onishi Y, et al. Non-small cell lung cancer: whole-body MR examination for M-stage assessment--utility for whole-body diffusion-weighted imaging compared with integrated FDG PET/CT. Radiology 2008;248:643-54.

8. Ohno Y, Koyama H, Yoshikawa T, et al. N stage disease in patients with non-small cell lung cancer: efficacy of quantitative and qualitative assessment with STIR turbo spin-echo imaging, diffusion-weighted MR imaging, and fluorodeoxyglucose PET/CT. Radiology 2011;261:605-15.

9. Coolen J, De Keyzer F, Nafteux P, et al. Malignant pleural disease: diagnosis by using diffusion-weighted and dynamic contrast-enhanced MR imaging--initial experience.

Radiology 2012;263:884-92. 
10. Zhang J, Cui LB, Tang X, et al. DW MRI at 3.0 T versus FDG PET/CT for detection of malignant pulmonary tumors. Int J Cancer 2014;134:606-611.

11. Mayerhoefer ME, Karanikas G, Kletter K, et al. Evaluation of diffusion-weighted MRI for pretherapeutic assessment and staging of lymphoma: results of a prospective study in 140 patients. Clin Cancer Res 2014;20:2984-93.

12. Deppen SA, Blume JD, Kensinger CD, et al. Accuracy of FDG-PET to diagnose lung cancer in areas with infectious lung disease: a meta-analysis. JAMA 2014;312:1227-36.

13. Shen G, Jia Z, Deng H. Apparent diffusion coefficient values of diffusion-weighted imaging for distinguishing focal pulmonary lesions and characterizing the subtype of lung cancer: a meta-analysis. Eur Radiol 2016;26:556-66.

14. Wu LM, Xu JR, Hua J, et al. Can diffusion-weighted imaging be used as a reliable sequence in the detection of malignant pulmonary nodules and masses? Magn Reson Imaging 2013;31:235-46.

15. Moher D, Liberati A, Tetzlaff J, et al. Preferred reporting items for systematic reviews and meta-analyses: the PRISMA statement. BMJ 2009;339:b2535.

16. Whiting PF, Rutjes AW, Westwood ME, et al. QUADAS-2: a revised tool for the quality assessment of diagnostic accuracy studies. Ann Intern Med 2011;155:529-36.

17. Deeks JJ, Altman DG. Diagnostic tests 4: likelihood ratios. BMJ 2004;329:168-9.

18. Simel DL, Samsa GP, Matchar DB. Likelihood ratios with confidence: sample size estimation for diagnostic test studies. J Clin Epidemiol 1991;44:763-70.

19. Simel DL, Bossuyt PM. Differences between univariate

Cite this article as: Chang N, Wang XH, Cui LB, Yin H, Jiang T, Chen FL, Liu LP, Zhang J. Diagnostic performance of diffusion-weighted magnetic resonance imaging in pulmonary malignant lesions: a meta-analysis. Transl Lung Cancer Res 2019;8(6):738-747. doi: 10.21037/tlcr.2019.10.08 and bivariate models for summarizing diagnostic accuracy may not be large. J Clin Epidemiol 2009;62:1292-300.

20. Deeks JJ, Macaskill P, Irwig L. The performance of tests of publication bias and other sample size effects in systematic reviews of diagnostic test accuracy was assessed. J Clin Epidemiol 2005;58:882-93.

21. van Houwelingen HC, Arends LR, Stijnen T. Advanced methods in meta-analysis: multivariate approach and metaregression. Stat Med 2002;21:589-624.

22. Centers for Medicare and Medicaid Services: Decision memo for positron emission tomography (FDG) for solid tumors (CAG-00181R4). Available online: http:// www.cms.gov/medicare-coverage-database/details/ncadecisionmemo.aspx? NCAId=26

23. Cancer Care Ontario: PET imaging in Ontario. Available online: http://www.cancercare.on.ca/ocs/clinicalprogs/ imaging/pet

24. Torre LA, Bray F, Siegel RL, et al. Global cancer statistics, 2012. CA Cancer J Clin 2015;65:87-108.

25. Siegel R, Ma J, Zou Z, et al. Cancer statistics, 2014. CA Cancer J Clin 2014;64:9-29.

26. Ye XH, Gao JY, Yang ZH, et al. Apparent diffusion coefficient reproducibility of the pancreas measured at different MR scanners using diffusion-weighted imaging. J Magn Reson Imaging 2014;40:1375-81.

27. Huang B, Law MW, Khong PL. Whole-body PET/CT scanning: estimation of radiation dose and cancer risk. Radiology 2009;251:166-174.

28. National Research Council. Health risks from exposure to low levels of ionizing radiation: BEIR VII phase 2 . Washington DTNAP, 2006. 
Table S1 Baseline characteristics of the included studies

\begin{tabular}{|c|c|c|c|c|c|c|c|c|c|c|c|c|}
\hline Study & Country & $\begin{array}{l}\text { Patient } \\
\text { number }\end{array}$ & $\begin{array}{l}\text { Lesion } \\
\text { number }\end{array}$ & Man/women & Age & Design & $\begin{array}{l}\text { Consecutive } \\
\text { enrollment }\end{array}$ & $\begin{array}{l}\text { Magnetic field } \\
\text { strength, } T\end{array}$ & $\mathrm{~b}$ value, $\mathrm{s} / \mathrm{mm}^{2}$ & Modality & Analytical method & Lesion size \\
\hline Chen W 2010 (29) & China & 56 & 7 & $35 / 21$ & 51 & $P$ & Yes & 1.5 & 1,000 & Only & Qualitative & $>2 \mathrm{~cm}$ \\
\hline Cai C 2011 (30) & China & 133 & 97 & $77 / 56$ & 55 & $\mathrm{R}$ & ND & 1.5 & 600 & Combined & Quantitative & $<2 \mathrm{~cm}$ \\
\hline Chen A 2010 (31) & China & 58 & 58 & $38 / 20$ & 55 & $P$ & ND & 1.5 & $300,600,900$ & Combined & Quantitative & $<2 \mathrm{~cm}$ \\
\hline Coolen J 2012 (32) & Belgium & 80 & 80 & ND & ND & $P$ & Yes & 3.0 & ND & Only & Quantitative & ND \\
\hline Coolen J 2014 (33) & Belgium & 54 & 54 & $36 / 18$ & 61 & $P$ & Yes & 3.0 & $50,500,750,1,000$ & Combined & Quantitative & $>2 \mathrm{~cm}$ \\
\hline Das SK 2017 (34) & China & 32 & 27 & ND & ND & $P$ & Yes & 3.0 & $500,1,000$ & Only & Quantitative & ND \\
\hline Deng QM 2012 (35) & China & 71 & 71 & $46 / 25$ & 52 & $\mathrm{R}$ & ND & 1.5 & $300,500,800$ & Only & Quantitative & $<2 \mathrm{~cm}$ \\
\hline Gümüştaş S 2012 (36) & Turkey & 67 & 67 & $49 / 17$ & 64 & $P$ & Yes & 1.5 & $500,1,000$ & Only & Quantitative & $>2 \mathrm{~cm}$ \\
\hline Guo MT 2018 (37) & China & 46 & 48 & $33 / 13$ & ND & $P$ & Yes & 3.0 & 1,000 & Only & Quantitative & $<2 \mathrm{~cm}$ \\
\hline Jao ZY 2019 (38) & China & 96 & 96 & $43 / 53$ & ND & $P$ & Yes & 3.0 & $600,800,1,000$ & Combined & Quantitative & ND \\
\hline Jouvet JC 2014 (39) & France & 37 & 45 & ND & ND & $P$ & ND & 1.5 & 600 & Combined & Qualitative & $<2 \mathrm{~cm}$ \\
\hline Koyama H 2010 (40) & Japan & 32 & 33 & $14 / 18$ & 65 & $P$ & Yes & 1.5 & 1,000 & Only & Qualitative & $>2 \mathrm{~cm}$ \\
\hline Koyama H 2015 (41) & Japan & 32 & 36 & $20 / 12$ & 68 & $\mathrm{P}$ & Yes & 1.5 & $500,1,000$ & Combined & Qualitative & $<2 \mathrm{~cm}$ \\
\hline Laurent V 2010 (42) & France & 35 & 14 & ND & ND & $P$ & Yes & 1.5 & 600 & Combined & Qualitative & $<2 \mathrm{~cm}$ \\
\hline Li W 2011 (43) & China & 116 & 120 & $69 / 47$ & 58 & ND & ND & 3.0 & $200,500,800,1,000$ & Only & Quantitative & $>2 \mathrm{~cm}$ \\
\hline Liu H 2010 (44) & China & 62 & 66 & $38 / 24$ & 58 & $\mathrm{P}$ & Yes & 1.5 & 500 & Only & Quantitative & $>2 \mathrm{~cm}$ \\
\hline Mori T 2008 (45) & Japan & 104 & 140 & $55 / 49$ & 68 & $\mathrm{P}$ & ND & 1.5 & 1,000 & Combined & Quantitative & $<2 \mathrm{~cm}$ \\
\hline Nomori H 2015 (46) & Japan & 77 & 87 & ND & ND & $P$ & ND & 1.5 & 800 & Combined & Qualitative & $<2 \mathrm{~cm}$ \\
\hline Ohba Y 2011 (47) & Japan & 58 & 76 & ND & ND & $P$ & ND & 1.53 .0 & 1,000 & Combined & Quantitative & $>2 \mathrm{~cm}$ \\
\hline Ohba Y 2009 (48) & Japan & 110 & 124 & $56 / 54$ & 68 & $\mathrm{R}$ & ND & 1.5 & 1,000 & Only & Quantitative & $<2 \mathrm{~cm}$ \\
\hline Ohno Y 2019 (49) & Japan & 57 & 71 & $38 / 19$ & ND & $P$ & Yes & 3.0 & ND & Combined & Qualitative & ND \\
\hline Ohno Y 2008 (7) & Japan & 203 & 51 & $109 / 94$ & 72 & $P$ & Yes & 1.5 & 1,000 & Combined & Qualitative & ND \\
\hline Regier M 2011 (50) & Germany & 20 & 71 & $10 / 10$ & 66 & $\mathrm{P}$ & Yes & 1.5 & 500 & Combined & Qualitative & $<2 \mathrm{~cm}$ \\
\hline Satoh S 2008 (51) & Japan & 51 & 54 & $37 / 14$ & 66 & $P$ & Yes & 1.5 & 1,000 & Combined & Qualitative & $>2 \mathrm{~cm}$ \\
\hline Sommer G 2012 (52) & Switzerland & 33 & 31 & $24 / 9$ & 66 & $P$ & ND & 1.5 & 800 & Combined & Qualitative & $>2 \mathrm{~cm}$ \\
\hline Tanaka R 2006 (53) & Japan & 43 & 43 & $22 / 21$ & 66 & ND & ND & 1.5 & 1,000 & Combined & Qualitative & $>2 \mathrm{~cm}$ \\
\hline Tanaka R 2007 (54) & Japan & 45 & 45 & $19 / 26$ & 68 & ND & ND & 1.5 & 1,000 & Combined & Qualitative & $>2 \mathrm{~cm}$ \\
\hline Tanaka R 2009 (55) & Japan & 46 & 72 & $18 / 28$ & 67 & $P$ & Yes & 1.5 & 1,000 & Only & Qualitative & $<2 \mathrm{~cm}$ \\
\hline Tondo F 2011 (56) & Italy & 34 & 34 & $25 / 9$ & 59 & ND & ND & 1.5 & $500,1,000$ & Combined & Quantitative & $<2 \mathrm{~cm}$ \\
\hline Uto T 2009 (57) & Japan & 28 & 28 & $22 / 6$ & 64 & ND & ND & 1.5 & 1,000 & Combined & Qualitative & $>2 \mathrm{~cm}$ \\
\hline Wang W 2010 (58) & China & 105 & 105 & $58 / 47$ & 57 & ND & ND & 1.5 & 1,000 & Combined & Qualitative & $>2 \mathrm{~cm}$ \\
\hline Wang MJ 2011 (59) & China & 56 & 56 & $39 / 17$ & 58 & $P$ & ND & 3.0 & $300,500,700,900$ & Combined & Qualitative & $>2 \mathrm{~cm}$ \\
\hline Wu HW 2008 (60) & China & 61 & 61 & $42 / 19$ & 58 & ND & ND & 3.0 & 500 & Combined & Quantitative & $>2 \mathrm{~cm}$ \\
\hline Yang TH 2008 (61) & China & 13 & 24 & ND & ND & ND & ND & 1.5 & $400,500,600$ & Only & Qualitative & ND \\
\hline Zhang J 2014 (10) & China & 113 & 113 & $67 / 46$ & 59 & $P$ & Yes & 3.0 & 1,000 & Combined & Quantitative + qualitative & $<2 \mathrm{~cm}$ \\
\hline Zhang YJ 2018 (62) & China & 50 & 50 & $28 / 22$ & 61 & $\mathrm{R}$ & ND & 1.5 & 800 & Combined & Quantitative & $>2 \mathrm{~cm}$ \\
\hline Zhou SC 2016 (63) & China & 56 & 56 & $41 / 15$ & 52 & $P$ & Yes & 1.5 & 500 & Only & Quantitative & ND \\
\hline
\end{tabular}

P, prospective; R, retrospective; ND, not documented; only, DWI; combined, DWI combined with other sequence. 


\section{References}

29. Chen W, Jian W, Li HT, et al. Whole-body diffusionweighted imaging vs. FDG-PET for the detection of nonsmall-cell lung cancer. How do they measure up? Magn Reson Imaging 2010;28:613-20.

30. Cai C, Zhao S, Lin L, et al. The value of MR difusion weighted imaging with STIR-EPI sequence for diferentiating benign from malignant pulmonary nodules. J Pract Med Imaging 2011;12:358-61.

31. Chen A, Li H, Liu S, et al. Differentiation of Malignant and Benign Pulmonary Lesions with Diffusion-weighted Imaging. Chest lmaging 2010;16:206-10.

32. Coolen J, De Keyzer F, De Leyn P, et al. DiffusionWeighted MRI Can Improve Preoperative Lung Cancer Diagnosis: The Necessity of a Meticulous ADC Curve Evaluation. Am J Roentgenol 2012;198(5).

33. Coolen J, Vansteenkiste J, De Keyzer F, et al. Characterisation of solitary pulmonary lesions combining visual perfusion and quantitative diffusion MR imaging. Eur Radiol 2014;24:531-41.

34. Das SK, Lin JZ, Yang DJ, et al. Non-Gaussian diffusion imaging for malignant and benign pulmonary nodule differentiation: a preliminary study. Acta Radiol 2017;58:19-26.

35. Deng QM, Qiu WJ, Zhou ZP, et al. Value of b value in differential diagnosis of benign and malignant lung lesions with DWI. Chin J Med Imaging Technol 2012;28:1537-40.

36. Gümüştaş S, Inan N, Akansel G, et al. Differentiation of malignant and benign lung lesions with diffusion-weighted MR imaging. Radiol Oncol 2012;46:106-13.

37. Guo MT, Wang YM, Liu YX, et al. Clinical value of 3.0T multiple b value magnetic resonance diffusion-weighted imaging (DWI) in the diagnosis of benign and malignant lung diseases. Journal of Imaging Research and Medical Applications 2018;2:154-6.

38. Jiao ZY, Hu CH, Du F, et al. Value of Dynamic ContrastEnhanced MRI Combined with Multiple b-Value Diffusion-Weighted Imaging for Differentiation of Benign and Malignant Lung Lesions. Journal of Clinical Radiology 2019;38:239-43.

39. Jouvet JC, Thomas L, Thomson V, et al. Whole-body MRI with diffusion-weighted sequences compared with 18 FDG PET-CT, CT and superficial lymph node ultrasonography in the staging of advanced cutaneous melanoma: a prospective study. J Eur Acad Dermatol Venereol 2014;28:176-85.
40. Koyama H, Ohno Y, Aoyama N, et al. Comparison of STIR turbo SE imaging and diffusion-weighted imaging of the lung: capability for detection and subtype classification of pulmonary adenocarcinomas. Eur Radiol 2010;20:790-800.

41. Koyama H, Ohno Y, Seki S, et al. Value of diffusionweighted MR imaging using various parameters for assessment and characterization of solitary pulmonary nodules. Eur J Radiol 2015;84:509-15.

42. Laurent V, Trausch G, Bruot O, et al. Comparative study of two whole-body imaging techniques in the case of melanoma metastases: advantages of multi-contrast MRI examination including a diffusion-weighted sequence in comparison with PET-CT. Eur J Radiol 2010;75:376-83.

43. Li W, Li D, Liu H, et al. 3.0T MR diffusion-weighted imaging: Evaluating diagnosis potency of pulmonary solid benign lesions and malignant tumors and optimizing $b$ value. Zhongguo Fei Ai Za Zhi 2011;14:853-7.

44. Liu H, Liu Y, Yu T, et al. Usefulness of diffusion-weighted MR imaging in the evaluation of pulmonary lesions. Eur Radiol 2010;20:807-15.

45. Mori T, Nomori H, Ikeda K, et al. Diffusion-weighted magnetic resonance imaging for diagnosing malignant pulmonary nodules/masses: comparison with positron emission tomography. J Thorac Oncol 2008;3:358-64.

46. Nomori H, Cong Y, Sugimura H, et al. Comparing diffusion-weighted imaging and positron emission tomography for pulmonary nodules measuring from 1 to 3 cm in size. Surg Today 2015;45:1535-41.

47. Ohba Y, Nomori H, Mori T, et al. Diffusion-weighted magnetic resonance for pulmonary nodules: 1.5 vs. 3 Tesla. Asian Cardiovasc Thorac Ann 2011;19:108-14.

48. Ohba Y, Nomori H, Mori T, et al. Is diffusion-weighted magnetic resonance imaging superior to positron emission tomography with fludeoxyglucose F 18 in imaging non-small cell lung cancer? J Thorac Cardiovasc Surg 2009;138:439-45.

49. Ohno Y, Fujisawa Y, Yui M, et al. Solitary pulmonary nodule: Comparison of quantitative capability for differentiation and management among dynamic CEperfusion MRI at 3T system, dynamic CE-perfusion ADCT and FDG-PET/CT. Eur J Radiol 2019;115:22-30.

50. Regier M, Schwarz D, Henes FO, et al. Diffusionweighted MR-imaging for the detection of pulmonary nodules at 1.5 Tesla: intraindividual comparison with multidetector computed tomography. J Med Imaging Radiat Oncol 2011;55:266-74.

51. Satoh S, Kitazume Y, Ohdama S, et al. Can malignant and 
benign pulmonary nodules be differentiated with diffusionweighted MRI? AJR Am J Roentgenol 2008;191:464-70.

52. Sommer G, Wiese M, Winter L, et al. Preoperative staging of non-small-cell lung cancer: comparison of wholebody diffusion-weighted magnetic resonance imaging and 18F-fluorodeoxyglucose-positron emission tomography/ computed tomography. Eur Radiol 2012;22:2859-67.

53. Tanaka R, Horikoshi H, Nakazato Y, et al. MR imaging in peripheral lung lesions. Jpn J Lung Cancer 2006;46:199-205.

54. Tanaka R, Horikoshi H, Nakazato Y, et al. Magnetic resonance imaging in peripheral lung cancer. Jpn J Lung Cancer 2007;47:13-20.

55. Tanaka R, Horikoshi H, Nakazato Y, et al. Magnetic resonance imaging in peripheral lung adenocarcinoma: correlation with histopathologic features. J Thorac Imaging 2009;24:4-9.

56. Tondo F, Saponaro A, Stecco A, et al. Role of diffusionweighted imaging in the differential diagnosis of benign and malignant lesions of the chest-mediastinum. Radiol Med 2011;116:720-33.

57. Uto T, Takehara Y, Nakamura Y, et al. Higher sensitivity and specificity for diffusion-weighted imaging of malignant lung lesions without apparent diffusion coefficient quantification. Radiology 2009;252:247-54.

58. Wang $\mathrm{W}, \mathrm{Xu} \mathrm{JZ}$, Zhang T, et al. Diffusion-weighted magnetic resonance imaging with short $\mathrm{T} 1$ inversion recovery-echo planar imaging combined with dual-head coincidence single photon emission computed tomography for diagnosing solitary pulmonary nodule. Chin Med J (Engl) 2010;123:3717-21.

59. Wang MJ, Zhang W, Zhang N, et al. Diagnostic Value of 3.0T Magnetic Resonance Diffusion-weighted Imaging Benign and Malignant Lung Tumors. J Nanchang University (Med Sci) 2011;51:14-9.

60. Wu HW, Cheng JJ, Xu JR, et al. The preliminary study of MR difusion weighted imaging with background body simlal suppression on pulmonary disease. Chin J Radiol 2008;42:56-9.

61. Yang TH, Lin JZ, Wang X, et al. Preliminary study of feasibility of whole body diffusion weighted imaging in diagnosis of metastasis of tumor. Chin Med Sci J 2008;23:187-92.

62. Zhang YJ, Cui AN, Li LX, et al. Clinical significance of spiral CT and DWI in differential diagnosis of benign and malignant lung diseases. Journal of Imaging Research and Medical Applications 2018;2:101-3.

63. Zhou SC, Xia LM, Wu W, et al. Single b-value diffusion weighted imaging in the diagnosis of pulmonary benign and malignant lesion. Radiol Prat 2016;31:728-33. 